\title{
Structured Reporting in Cross-Sectional Imaging of the Heart: Reporting Templates for CMR Imaging of Cardiomyopathies (Myocarditis, Dilated Cardiomyopathy, Hypertrophic
} Cardiomyopathy, Arrhythmogenic Right Ventricular Cardiomyopathy and Siderosis)

\section{Strukturierte Befundung in der Schnittbilddiagnostik des Herzens: Befundvorlagen für die MRT bei Kardiomyopathien (Myokarditis, dilatative Kardiomyopathie, hypertrophe Kardiomyopathie, arrhythmogene rechtsventrikuläre Kardiomyopathie und Siderose)}

\section{Authors}

Alexander Christian Bunck'1, Bettina Baeßler ${ }^{1,2}$, Christian Ritter ${ }^{3}$, Jan Robert Kröger ${ }^{1}$, Thorsten Persigehl', Daniel Pinto dos Santos ${ }^{1}$, Michael Steinmetz ${ }^{4}$, Adelheid Niehaus ${ }^{5}$, Fabian Bamberg ${ }^{6}$, Meinrad Beer ${ }^{7}$, Sebastian Ley ${ }^{8}$, Klaus Tiemann ${ }^{9}$, Philipp Beerbaum ${ }^{10}$, David Maintz ${ }^{1}$, Joachim Lotz ${ }^{3}$

Affiliations

1 Department of Diagnostic and Interventional Radiology, University of Cologne, Faculty of Medicine and University Hospital Cologne, Cologne, Germany

2 Institute of Diagnostic and Interventional Radiology, University Hospital Zurich, University of Zurich, Zurich, Switzerland

3 Department of Radiology, University Medical Center Göttingen, Göttingen, Germany

4 Department of Pediatric Cardiology and Intensive Care Medicine, University Medical Center Göttingen, Göttingen, Germany

5 Department of Thoracic, Cardiac and Vascular Surgery, University Medical Center Göttingen, Göttingen, Germany

6 Department of Diagnostic and Interventional Radiology, Medical Center - University of Freiburg, Faculty of Medicine, University of Freiburg, Germany

7 Department of Radiology, University-Hospital Ulm, Germany

8 Department of Radiology, Internistisches Klinikum München-Süd, München, Germany

9 Clinic of Internal Medicine I, Klinikum rechts der Isar der Technischen Universität München, München, Germany

10 Department of Pediatric Cardiology and Intensive Care Medicine, Hannover Medical School, Hannover, Germany

Key words

structured reporting, heart, cardiomyopathy, CMR

received 17.12 .2018

accepted 01.08.2019
Bibliography

DOI https://doi.org/10.1055/a-0998-4116

Published online: 9.10.2019

Fortschr Röntgenstr 2020; 192: 27-37

(C) Georg Thieme Verlag KG, Stuttgart · New York ISSN 1438-9029

Correspondence

Priv.-Doz. Dr. med. Alexander Christian Bunck

Department of Diagnostic and Interventional Radiology, University of Cologne, Faculty of Medicine and University Hospital Cologne, Kerpener Straße 62, 50967 Cologne, Germany

Tel.: ++ 49/2 21/47882035

Fax: $++49 / 221 / 4784213$

alexander.bunck@uk-koeln.de

\section{ABSTRACT}

Backround Structured reports have numerous benefits through standardizing the way imaging findings are reported and communicated. Nevertheless, the adoption of structured reports in everyday radiological practice is still limited. In view of the irrefutable benefits, various national and international radiological societies have started initiatives which aim at promoting a broader use of structured reports. Up to now, no consented templates in German language existed for the reporting of cross-sectional imaging studies of the heart.

Method Upon invitation of the working group for Cardiovascular Imaging of the German Society of Radiology a panel of radiologists, cardiologists, pediatric cardiologists and cardiothoracic surgeons, experts on the field of cardiovascular imaging and structured reporting, met for two interdisciplinary consensus meetings at the University Hospital Cologne in 2018. The aim of these meetings was to develop and agree 
on templates for the reporting of MR and CT studies of various cardiovascular disease entities.

Results During the meetings the panel of experts developed and reached consensus on 11 different templates for the structured reporting of the following: myocarditis, dilated cardiomyopathy, hypertrophic (obstructive) cardiomyopathy, arrythmogenic right ventricular cardiomyopathy, siderosis, ischemia and vitality imaging, tetralogy of Fallot, aortic coarctation, coronary CT and CT for Transcatheter Aortic Valve Implantation (TAVI) planning. The first five templates are presented in this publication and are currently being transferred to a HTML 5/IHR MRRT compatible format. Subsequently, the templates will be made available for free use on the website www.befundung.drg.de.

Conclusion For the first time, consented templates in German language for the structured reporting of cross-sectional imaging studies of the heart are presented. These templates are aimed at providing a constant level of high reporting quality and increasing the efficiency of the generation and communication of imaging reports.

\section{Key points:}

- Structured reporting offers numerous benefits by standardizing generation and communication of imaging reports.

- For the first time templates in German language for the structured reporting of CMR imaging studies of cardiomyopathies are presented

- These templates will be made available on the website www.befundung.drg.de and can be commented via agit-sr@googlegroups.com.

\section{Citation Format}

- Bunck AC, Baeßler B, Ritter C et al. Structured Reporting in Cross-Sectional Imaging of the Heart: Reporting Templates for CMR Imaging of Cardiomyopathies (Myocarditis, Dilated Cardiomyopathy, Hypertrophic Cardiomyopathy, Arrhythmogenic Right Ventricular cardiomyopathy and Siderosis). Fortschr Röntgenstr 2020; 192: 27-37

\section{ZUSAMMENFASSUNG}

Hintergrund Strukturierte Befundung bietet viele Vorteile in der Standardisierung der Befunderstellung und -kommunikation und bildet dennoch bisher die Ausnahme im radiologischen Alltag. Den Einsatz der strukturierten Befundung zu fördern ist daher erklärtes Ziel nationaler und internationaler radiologischer Gesellschaften. Bisher existierten für den deutschsprachigen Raum keine konsentierten Befundvorlagen auf dem Gebiet der kardialen Schnittbilddiagnostik.

Methode Auf Einladung der AG Herz- und Gefäßdiagnostik der Deutschen Röntgengesellschaft fanden an der Uniklinik Köln im Jahr 2018 zwei interdisziplinäre Konsensus-Treffen zwischen Experten der kardiovaskulären Bildgebung und strukturierten Befundung aus den Fachrichtungen Radiologie, Kardiologie, Kinderkardiologie und Thorax-, Herz- und Gefäßchirurgie statt. Ziel der Treffen war es, im interdisziplinären Diskurs Befundvorlagen zur bildgebenden CT- und MRDiagnostik verschiedener Krankheitsbilder und Untersuchungen des Herzens gemeinsam zu erarbeiten.

Ergebnisse Im Rahmen der beiden Treffen wurden Vorlagen zu folgenden Krankheitsbildern und Untersuchungen entwickelt und im Konsens verabschiedet: Myokarditis, dilatative Kardiomyopathie, hypertrophe (obstruktive) Kardiomyopathie, arrhythmogene rechtsventrikuläre Kardiomyopathie (ARVC), Siderose, Ischämie- und Vitalitätsdiagnostik, Fallot' sche Tetralogie, Aortenisthmusstenose, Koronar-CT und TAVI-CT. Die 5 erstgenannten Vorlagen sind Gegenstand der vorliegenden Publikation und werden aktuell von der AG für Informationstechnologie (AGIT) der DRG in ein HTML-5/IHEMRRT-konformes Format überführt. Anschließend werden die Vorlagen auf der Seite www.befundung.drg.de zur freien Nutzung bereitgestellt werden.

Schlussfolgerung Im Rahmen der vorliegenden Publikation werden erstmals interdisziplinär konsentierte deutschsprachige Befundvorlagen für die Schnittbilddiagnostik des Herzens vorgeschlagen. Der Einsatz der erarbeiteten Vorlagen soll helfen, für eine gleichbleibend hohe Befundungsqualität zu sorgen und die Effizienz der Befunderstellung und -kommunikation zu steigern.

\section{Structured Reporting - Background}

To date, the majority of radiological reports are written in the form of non-standardized free text. A number of studies have demonstrated that the use of standardized reporting templates offer advantages for both the reporting and communication of findings [1-5]. Thematic structure, pre-formulated text modules, categorized diagnoses and uniform terminology can increase the time efficiency in the preparation of reports, while standardization can help ensure consistent quality with respect to comprehensibility, clarity, completeness and clinical relevance of the reports [6, 7].

To take this into account, RSNA launched two initiatives with the "RadLex Initiative" in 2005 and the "Radiology Reporting Initiative" in 2008 with the aim of harmonizing the terminology used in radiology, promoting the use of structured reporting templates and thus increasing the overall quality of radiological reports [8]. RadLex serves as a standardized ontology and encyclopedia of radiological terms and was recently translated into German by the German Radiological Society (DRG) in cooperation with RSNA [9]. Likewise, the European Radiological Society (ESR) has also joined the initiative for structured reporting and has established the goal of developing reports in different national languages [6]. Existing reporting templates of RSNA and ESR are made available on the open platform www.radreport.org; they are thematically organized, freely accessible in the HTML/IHE MRRT format [10] and available for general use.

In a joint statement published in 2009, numerous organizations in the field of cardiovascular medicine also clearly expressed 
their support for the use of structured reporting [7]. The initial basic recommendations on the structure and content of reports on MR cardiovascular examinations were published in the same year by the Society of Cardiovascular Magnetic Resonance (SCMR) [11].

However, the number of templates currently available on the www.radreport.org platform in the area of cardiac diagnostics is still limited, with respect to both content and thematics. Up to date, no German templates available.

In Germany, the board of directors of the DRG has made the promotion of structured reporting one of that organization's central projects for the coming years [12]. The working groups of the various specialty areas have been tasked with defining reporting templates and their medical content. Technical support for this project will be provided by the Information Technology working group of the DRG. After formatting in HTML 5/IHE MRRT, the developed templates will be made freely available on the DRG homepage www.befundung.drg.de.

\section{Initiative for the Development of Reporting Templates for Cardiac Cross-sectional Imaging Diagnostics}

Against this background, board members and representatives of the working group for Cardiac and Vascular Diagnostics of the DRG agreed during the 10th Cardiodiagnostic Days in Leipzig, Germany, on the joint development of proposals for reporting templates for cross-sectional imaging of the heart. At the invitation of the working group, two consensus meetings were held at the University Hospital Cologne, Germany in 2018 with the participation of the listed authors. During these meetings experts from the fields of cardiovascular diagnostics and structured reporting jointly developed a total of 11 reporting templates for various examination protocols and clinical pathologies in the field of cardiac MRI and CT diagnostics. The authors clearly preferred a systematic orientation towards modalities, examination protocols and clinical pathologies rather than a generic approach with the elaboration of only a few general reporting templates, since usually the examination protocols in MRI and CT diagnostics are also oriented towards specific suspected diagnoses and clinical issues.

In total, 11 templates were developed for the following clinical pathologies and examination protocols: myocarditis, dilatative cardiomyopathy, hypertrophic (obstructive) cardiomyopathy, arrhythmogenic right ventricular cardiomyopathy (ARVC), siderosis, ischemia and viability diagnostics, tetralogy of Fallot, aortic coarctation, coronary CT, TAVI CT.

All templates were approved by consensus of all involved authors. The templates do not contain any recommendations regarding examination protocols. Recommendations on this matter, as well as on image interpretation and quantitative analysis can be found in international guidelines, such as those of the SCMR [13-16].

\section{Report Contents}

Important, integral components of a report include clinical information on the patient, the justifying indication and clinical question derived from this, as well as the date and time of the examination; these should be presented first in the template [11].

The reporting templates presented in this publication comprise the thematic complex of cardiomyopathies including secondary forms of myocardial functional impairment as a result of cardiac siderosis and myocarditis.

The contents of the templates are based on the recommendations of the SCMR [11], current clinical practice, the present state of scientific knowledge [17] as well as in the case of ARVD and myocarditis on internationally published diagnostic standards. These are laid down in the modified Task Force Criteria for ARVD published in 2010 and in the Lake Louise Criteria revised in 2018 (Lake Louise Criteria II) [18-20]. The terminology reflects that used by RadLex in its current German-language version (www. radlex.org).

Each of the reporting templates is divided into the sections Technique, Patient Characteristics, the actual report sections Morphology, Functional Analysis, Tissue Characterization and Additional Findings as well as the Conclusion. In the chosen approach, a distinction is made between a "General" part and a "Specific" part.

The "General" part of the report is intended to precede a "Specific" part to be selected. The selection of the specific part depends mainly on the case history and the related clinical question or, in individual cases, also on the findings identified on image analysis and the derived (suspected) diagnosis. In the case of more complex findings, several specific parts can also be combined in a modular manner. Depending on the pathology, the general part can be adapted, as outlined in the explanations of the general part (items 4, 8, 9 and 14).

From the authors' point of view, this approach also reflects the way a radiologist would select and specify an appropiate MR imaging protocol in everyday clinical practice.

In the authors' opinion, the technical part should contain not only the field strength and list of the acquired sequences in the corresponding orientations but also the software used for image analysis, the source of the normal values for quantitative parameters as well as the quantity and type of contrast medium used.

In a 2015 review article, Kawel-Boehm et al. compiled the normal values for adults and children published in various studies which can serve as a reference for the quantitative values obtained with MRI of the heart [21]. However, assuming a representative control cohort, normal values can also be adapted to local conditions and new scientific findings. In this case, normal values should be chosen as a function of the age and sex of the patient. Particularly in the context of left ventricular volumetry, when selecting these standard values it must be taken into account whether the evaluation software assigns the papillary muscle and muscle trabecula to the ventricular cavum or the myocardial mass $[16,21]$.

The use and reporting of $\mathrm{T} 1$ and $\mathrm{T} 2$ relaxation times of the myocardium determined by mapping techniques and the derived parameter extracellular volume (ECV) in the findings section are 
regarded as optional by the authors and require normal values from the examiner's own representative control cohorts for the locally-used scanner and sequence type. In this respect, reference is made explicitly to the recommendations of the SCMR [22]. Despite known limitations, the use of mapping techniques is becoming increasingly important and widespread due to increasing evidence. This circumstance is also taken into account in the currently revised Lake Louise criteria [20] and supports, in the authors' opinion, the optional inclusion into the findings section.

The amount and type of contrast agent used, and in the case of first-pass perfusion images, the injection rate, can have a relevant influence on image contrast and image artifacts due to the different concentrations and relaxivities of the contrast agents as well as the field strength, so that these should be listed in the technique section [11, 23-25].

In the patient baseline profile section, gender, height, weight and body surface should be documented so that the quantitative values collected in the functional analysis can be normalized to the individual body surface.

The patient's ability to cooperate, heart function and rate can have a considerable influence on the image quality of the acquired MR images. Since reduced image quality can sometimes considerably limit the interpretation of the findings, the quality of the acquired images should be briefly commented on at the beginning of the findings section. If the image quality does not permit a conclusive assessment of the findings, this should also be mentioned in the final conclusion.

The found consensus on the presentation of functional parameters and specific morphological features varies depending on the significance for the respective clinical question or the pathology.

Regionally-limited abnormalities such as wall movement disorders should be assigned to the ventricle segment (apex, apical, midventricular, basal) and the myocardial segment (basal and midventricular: anterior, anterolateral, inferolateral, inferior, inferoseptal, anteroseptal or apical: anterior, lateral, inferior, septal) according to the 17-segment model of the American Heart Association - AHA [26]. The assignment of regional findings to the right ventricle can be done according to the 5-segment model of te Riele et al. (apex, inferior, marginal, anterior, RVOT (right ventricular outflow tract)) [27].
In contrast to normokinesia, the semi-quantitative assessment of the severity of regional wall movement disorders should be graded as follows: hyperkinesia (excessive contraction movement, i. e. increased systolic inward movement and increase in thickness); hypokinesia (reduced contraction movement, i. e. reduced systolic inward movement and increase in thickness); akinesia (lack of contraction movement), dyskinesia (outward movement of the affected segment in systole), aneurysm (protrusion of the affected segment in systole and diastole), tardokinesia (delayed contraction movement) and paradoxical wall movement (outward movement of the affected segment in systole, inward movement in diastole) $[11,16]$. In the case of focal changes of the signal intensity of the myocardium, localization in the myocardium (subendocardium, intramyocardium, subepicardium or transmural) and extent should also be described $[11,16]$

\section{Summary and Outlook}

The reporting templates presented here, developed in cooperation with clinical partners from cardiology, pediatric cardiology and cardiac surgery, are to be understood as a proposal of the DRG Cardiovascular working group for cardiac and vascular diagnostics, and are not to be considered binding or universally valid. The presentation of the 6 additional reporting templates developed among the authors is planned for follow-up publications. AGIT is currently converting the report forms presented here into HTML-5/IHE MRRT-compliant format and will make them available for free use on the www.befundung.drg.de website (for rights to use, see [28]) Comments on the proposed report forms can be sent at any time to agit-sr@googlegroups.com and will be forwarded by AGIT to the board of the working group for Cardiac and Vascular Diagnostics. The comments received, as well as new scientific evidence and guidelines, will be evaluated at regular intervals by the working group and any necessary changes will be incorporated by consensus into the reporting templates published on the DRG homepage www.befundung.drg.de. 


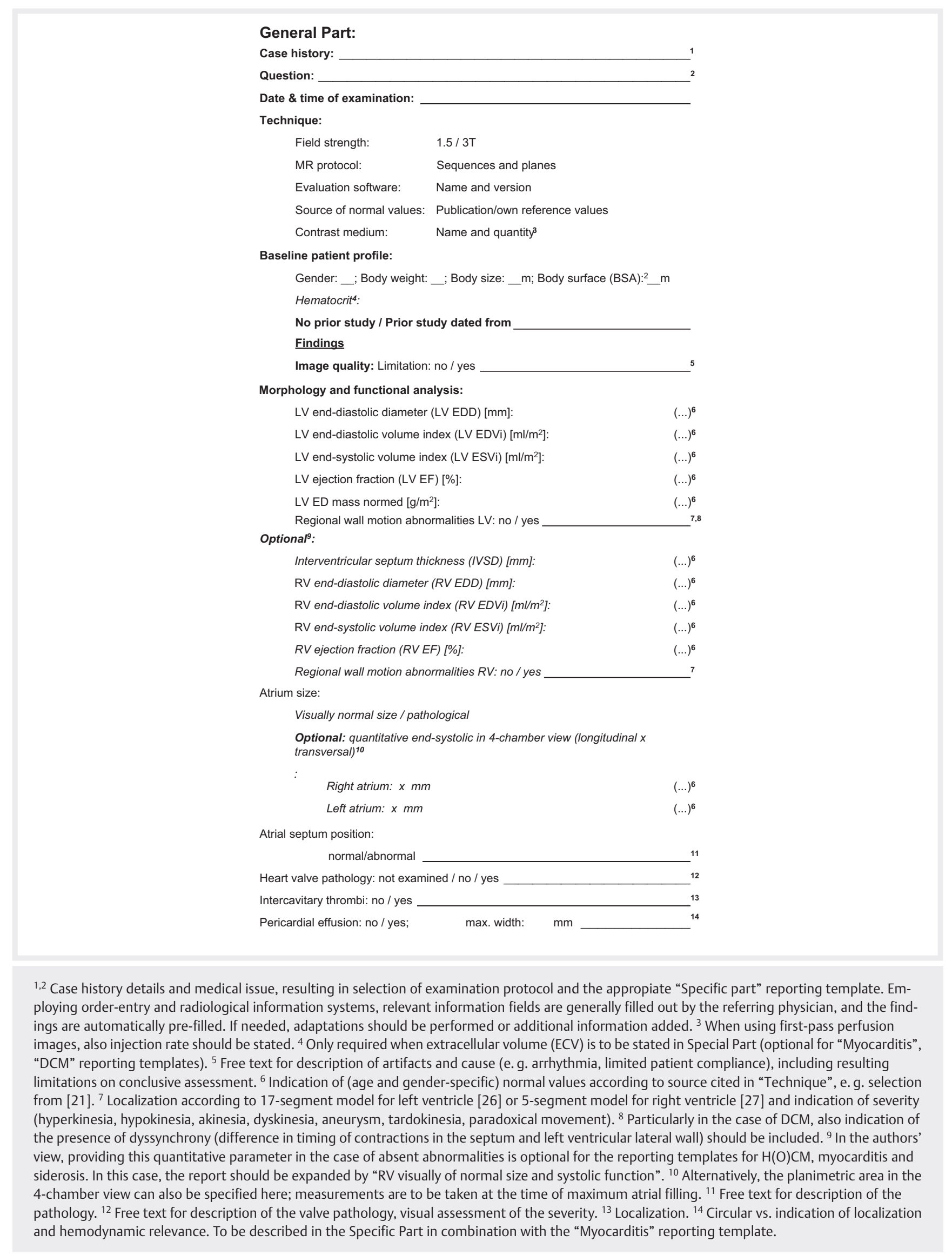




\section{Specific Part: "Myocarditis" reporting template}

\section{Tissue properties (modified according to Lake Louise Criteria II)1:}

Myocardial edema regional: no / yes

Myocardial edema global: no / yes

Late gadolinium enhancement (LGE) myocardium: no / yes

Late gadolinium enhancement (LGE) pericardium: no / yes

Pericardial effusion: no / max. width:

$\mathrm{mm}$

Pericardial thickening (>3 mm): no / yes

\section{Optional:}

T2 mapping [ms]:

T2 mapping native [ms]:

T2 mapping post CM [ms]:

ECV [\%]:

(with provision of sequence names in "Technique" section as well as

Feference values from own pool)

\section{Additional findings}

No / yes

\section{Conclusion:}

MRI findings consistent with myocarditis: no / possible / yes ${ }^{7}$

${ }^{1}$ The revised MR diagnostic criteria for myocarditis published in 2018 differentiate between T2- and T1-based primary myocardial criteria and supportive criteria (pericardial changes and systolic left ventricular myocardial dysfunction) [20]. A prerequisite for the diagnosis of myocarditis is the presence of at least one main criterion from the T2 and the T1 group. These also explicitly include findings derived from mapping sequences. As explained, the latter requires the existence of locally-established reference values for the particular scanner and sequence type [22]. ${ }^{2}$ Localization according to 17-segment model for left ventricle and indication of the distribution pattern (subendocardial, intramyocardial, subepicardial, transmural). ${ }^{3}$ Circular vs. indication of localization. ${ }^{4}$ In addition, indication of hemodynamic relevance according to [20]. ${ }^{5}$ In addition, indication of max. pericardial thickness according to [20]. ${ }^{6}$ Statement regarding distribution pattern of pathological values. Myocardial: diffuse vs. localized (localization according to 17-segment model, subendocardial, intramyocardial, subepicardial, transmural); Pericardial: circular vs. indication of localization [20]. ${ }^{7}$ Possible: presence of a T2 (T2-weighted imaging/T2 mapping) or a T1 (LGE/T1 mapping/ECV) diagnostic criterion; yes: presence of at least one T2 and at least one T1 diagnostic criterion [20]. ${ }^{8}$ Free text for secondary findings. If needed, also recommendations for follow-up, see also the white paper on cardiac MRI in myocarditis from 2009 [19]. 


\section{Reporting template "Dilatative Cardiomyopathy - DCM" \\ Tissue properties:}

Late gadolinium enhancement (LGE) myocardium: no / yes

\section{Optional:}

T2 mapping [ms]:

$(\ldots)^{\#}$

T2 mapping native [ms]:

$(\ldots)^{\#}$

T2 mapping post CM [ms]:

$(\ldots)^{\#}$

ECV $[\%]$ :

$(\ldots)^{\#}$

(with provision of sequence names in "Technique" section as well as

\#reference values from own pool)

\section{Additional findings}

No / yes

\section{Conclusion:}

MRI findings consistent with DCM: no / possible / yes²

3

${ }^{1}$ Localization according to 17-segment model and indication of the distribution pattern; a striped late enhancement in the center of the myocardium is indicative of a less favorable prognosis of DCM [29]. ${ }^{2}$ Possible: dilatation or functional impairment; yes: dilatation and functional impairment. ${ }^{3}$ Free text, e. g. for secondary findings, presumed etiology vs. idiopathic. 


\section{Hypertrophic (Obstructive) Cardiomyopathy - H(O)CM}

Max. LV wall thickness [mm]: 1, Localization

\section{2}

LVOT obstruction at rest ${ }^{3}$ :

no / yes

LVOT obstruction during Valsalva ${ }^{3,4}$ :

no / yes

SAM phenomenon of mitral valve:

no / yes

Associated eccentric mitral valve insufficiency: no / yes

\section{Tissue properties:}

Late gadolinium enhancement (LGE) myocardium: no / yes 5 Optional:

T2 mapping [ms]: $(\ldots)^{\#}$

T2 mapping native [ms]: $(\ldots)^{\#}$

T2 mapping post CM [ms]: $(\ldots)^{\#}$

ECV [\%]: $(\ldots)^{\#}$

(with provision of sequence names in "Technique" section as well as

\#reference values from own pool)

\section{Additional findings}

No / yes

\section{Conclusion:}

MRI findings consistent with HCM: no / possible / yes ${ }^{6}$

Obstruction: no / yes 8

${ }^{1}$ Measured in end-diastole. ${ }^{2}$ Localization according to 17 -segment model [26]. ${ }^{3}$ Visual detection of flow turbulence with signal drop due to dephasing in balanced Steady State Free Precession - bSSFP cine sequences. ${ }^{4}$ If needed, using real time images or phase-contrast flow measurement [13]. A Valsava maneuver can provoke LVOT obstruction and is regularly used in the course of echocardiogram examinations of HCM patients with suspected LVOT obstruction [30]. ${ }^{5}$ Localization according to 17 -segment model and indication of the distribution pattern; typical for $\mathrm{H}(\mathrm{O}) \mathrm{CM}$ is a somewhat faint intramyocardial basal anteroseptal and inferoseptal delayed enhancement as expression of fibrosis [31]. ${ }^{6}$ Regarding diagnosis definition, see e. g. Journal of Cardiovascular Magnetic Resonance 2012, 14: 17 [32]. ${ }^{7}$ For a description of involved wall segments and resulting morphological changes, see also Noureldin et al. Journal of Cardiovascular Magnetic Resonance 2012, 14:17 [32]. ${ }^{8}$ Free text for secondary findings. 


\title{
Reporting template "Arrhythmogenic Right Ventricular Cardiomyopathy/Dysplasia - ARVC/D"
}

\author{
(Micro) aneurysms RV wall: no / yes \\ Right ventricular wall thinning: no / yes \\ Dyssynchronous RV contraction:

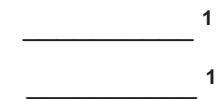 \\ no / yes \\ Optional: RVOT4 width in 3-chamber view*: \\ $\mathrm{mm} / \mathrm{m}^{2}$ (normal: $x x \mathrm{~mm} / \mathrm{m}^{2}$ ).
}

Tissue properties:

Late gadolinium enhancement (LGE) myocardium: no / yes 2

Evidence of fat signal in myocardium: no / yes

\section{Assessment according to Task Force criteria (revised 2010) \#:4}

Major criterion: no / yes

Minor criterion: no / yes

\section{Additional findings}

No / yes

\section{Conclusion:}

MRI indicative of ARVC: no / yes

- * Eur Heart J Cardiovasc Imaging. 2017 May 26. http://dx.doi.org/10.1093/ehjci/jex092 [33]. \# Eur Heart J. 2010;31:806-814. http://dx.doi.org/ 10.1093/eurheartj/ehq025 [18]. ' Localization. Typical locations are the "triangle of dysplasia": RV wall adjacent to the RV inflow and outflow path and the apex of the heart [18] or the basal anterior and inferior RV wall as well as the posterolateral LV wall [27]. ${ }^{2}$ Localization according to 17 segment model [26] or inclusion of the RV analogously to the 5-segment model [27] and indication of the distribution pattern (subendocardial/ intramyocardial/subepicardial/transmural). ${ }^{3}$ Localization: Typical features are focal, myocardial fatty degeneration or an "infiltrative", fingershaped fatty degeneration of the free RV wall progressing from the epicardium with myocardial wall thinning potentially associated with late gadolinium enhancement [34]; MR tomographic fat detection or late gadolinium enhancement are not part of the Task Force criteria [18]. ${ }^{4}$ Only one major or minor criterion can be derived from MR imaging alone [18]. The definitive diagnosis of ARVD requires the presence of at least 2 major criteria, 1 major criterion plus 2 minor criteria or 4 minor criteria from 6 different diagnostic categories; a definitive diagnosis cannot be made based solely on MR diagnostics. A "borderline" ARVD is based on evidence of one major plus one minor criterion or 3 minor criteria. A "possible" ARVD is based on evidence of one major criterion or two minor criteria. The MR criteria are defined as follows: Major criterion: Regional RV akinesia or dyskinesia or dyssynchronous RV contraction and presence of one of the following findings: Ratio of RV EDV to BSA $\geq 110 \mathrm{~mL} / \mathrm{m}^{2}(\mathrm{male}$ ) or $\geq 100 \mathrm{~mL} / \mathrm{m}^{2}$ (female) or RV ejection fraction $\leq 40 \%$. Minor criterion: Regional RV akinesia or dyskinesia or dyssynchronous RV contraction and Ratio of RV EDV to BSA $\geq 100$ to $<100 \mathrm{~mL} / \mathrm{m}^{2}$ (male) or $\geq 90$ to $<100 \mathrm{~mL} / \mathrm{m}^{2}$ (female) or RV ejection fraction $>40 \%$ to $\leq 45 \%$. ${ }^{5}$ Indication of presence of task force and non-task force criteria (ARVD-typical pattern of fat infiltration and/or non-ischemic LGE) [34], Free text for secondary findings.

Erratum on the article: Bunck AC, Baeßler B, Ritter C et al. Structured Reporting in Cross-Sectional Imaging of the Heart: Reporting Templates for CMR Imaging of Cardiomyopathies (Myocarditis, Dilated Cardiomyopathy, Hypertrophic Cardiomyopathy, Arrhythmogenic Right Ventricular cardiomyopathy and Siderosis). Fortschr Röntgenstr 2020; 192: 27-37

The name of the co-author Daniel Pinto dos Santos was completed on 6.11.2019. 


\section{"Siderosis" reporting template}

Quantitative tissue properties of the heart:

T2* mapping (septum) [ms]: (normal > $20 \mathrm{~ms}$ )

Optional:

T2 mapping native (septum) [ms]: $(\ldots)^{\#}$

T1 mapping native (septum) [ms]: $(\ldots)^{\#}$

(with provision of sequence names in "Technique" section as well as \#reference values from own pool)

Morphology of liver / upper abdomen:

Liver parenchyma:

Liver artery, veins, portal vein:

Bile ducts / gall bladder:

Spleen:

unremarkable /
unremarkable /
unremarkable /
unremarkable /

Lymph nodes: No suspicious lymph nodes / lymph node 1, approx._mm

Ascites: none / few / moderate / pronounced

Quantitative tissue properties of liver:

T2* mapping [ms]: (normal $>24 \mathrm{~ms}$ )

Optional:

T2 mapping native [ms]: $(\cdots)^{\#}$

T1 mapping native [ms]: $(\cdots)^{\#}$

(with provision of sequence names in "Technique" section as well as \#reference values from own pool)

Fat fraction: $\%$

\section{Additional findings}

No / yes

\section{Conclusion:}

Heart:

Based on 1.5 Tesla MRI signs of mild $\left(\mathrm{T}^{*}=15-20 \mathrm{~ms}\right) /$ moderate $\left(\mathrm{T}^{*}=10-14 \mathrm{~ms}\right) /$ severe $\left(\mathrm{T} 2^{*}<10 \mathrm{~ms}\right)$ cardiac iron overload / no evidence of cardiac iron overload $\left(\mathrm{T} 2^{*}>20 \mathrm{~ms}\right)$.

Unremarkable function of right and left ventricle / limited / moderate / severely limited LV/RV systolic function (EF_\%)

Liver:

Based on 1.5 Tesla MRI unremarkable ( $\left.\mathrm{T} 2^{*}>24 \mathrm{~ms}\right) /$ low $\left(\mathrm{T} 2^{*}<24 \mathrm{~ms}\right) /$ moderate $\left(\mathrm{T} 2^{*}\right.$ $<21 \mathrm{~ms}$ ) / pronounced $\left(\mathrm{T}^{*}<14 \mathrm{~ms}\right)$ iron accumulation. ${ }^{3}$

Secondary signs of mild / pronounced cirrhosis of the liver.

${ }^{1}$ If noticeable, description of pathology as free text. ${ }^{2}$ Graduation analogous to [22], according to these recommendations assessment for potential iron overload by means of T2* mapping should be performed at 1.5 Tesla only. ${ }^{3}$ Graduation analogous to [35]. ${ }^{4}$ Free text for secondary findings. 


\section{Conflict of Interest}

The authors declare that they have no conflict of interest.

\section{References}

[1] Schwartz LH, Panicek DM, Berk AR et al. Improving communication of diagnostic radiology findings through structured reporting. Radiology 2011; 260: 174-181

[2] Bosmans JM, Weyler JJ, De Schepper AM et al. The radiology report as seen by radiologists and referring clinicians: results of the COVER and ROVER surveys. Radiology 2011; 259: 184-195

[3] Franconeri A, Fang ], Carney B et al. Structured vs narrative reporting of pelvic MRI for fibroids: clarity and impact on treatment planning. Eur Radiol 2018; 28: 3009-3017

[4] Gassenmaier S, Armbruster M, Haasters F et al. Structured reporting of MRI of the shoulder - improvement of report quality? Eur Radiol 2017; 27: 4110-4119

[5] Patel A, Rockall A, Guthrie A et al. Can the completeness of radiological cancer staging reports be improved using proforma reporting? A prospective multicentre non-blinded interventional study across 21 centres in the UK. BMJ Open 2018; 8: e018499

[6] European Society of Radiology (ESR). ESR paper on structured reporting in radiology. Insights Imaging Februar 2018; 9: 1-7

[7] Douglas PS, Hendel RC, Cummings JE et al. ACCF/ACR/AHA/ASE/ASNC/ HRS/NASCI/RSNA/SAIP/SCAI/SCCT/SCMR 2008 Health Policy Statement on Structured Reporting in Cardiovascular Imaging. J Am Coll Cardiol 2009; 53: 76-90

[8] Morgan TA, Helibrun ME, Kahn CE. Reporting Initiative of the Radiological Society of North America: Progress and New Directions. Radiology 2014; 273: 642-645

[9] Deutschsprachige RadLex-Version veröffentlicht [Internet]. 2018 Mai. Verfügbar unter: https://www.drg.de/de-DE/4291/radlex-deutsch/

[10] IHE Radiology Technical Committee. IHE Radiology Technical Framework Supplement - Management of Radiology Report Templates (MRRT), Rev. 1.7 - Trial Implementation [Internet]. Verfügbar unter: https://www.ihe.net/uploadedFiles/Documents/Radiology/ IHE_RAD_SuppI_MRRT.pdf

[11] Hundley WG, Bluemke D, Bogaert JG et al. Society for Cardiovascular Magnetic Resonance guidelines for reporting cardiovascular magnetic resonance examinations. J Cardiovasc Magn Reson 2009; 11: 5

[12] Pinto dos Santos D, Hempel JM, Mildenberger $P$ et al. Structured Reporting in Clinical Routine. RöFo - Fortschritte Auf Dem Geb Röntgenstrahlen Bildgeb Verfahr [Internet] 13. August 2018 [zitiert 9. Dezember 2018]. Verfügbar unter: http://www.thieme-connect.de/DOI/ DOI:10.1055/a-0636-3851

[13] Kramer CM, Barkhausen J, Flamm SD et al. Standardized cardiovascular magnetic resonance (CMR) protocols 2013 update. J Cardiovasc Magn Reson 2013; 15: 91

[14] Kramer CM, Barkhausen J, Flamm SD et al. Standardized cardiovascular magnetic resonance imaging (CMR) protocols, society for cardiovascular magnetic resonance: board of trustees task force on standardized protocols. J Cardiovasc Magn Reson 2008; 10: 35

[15] Fratz S, Chung T, Greil GF et al. Guidelines and protocols for cardiovascular magnetic resonance in children and adults with congenital heart disease: SCMR expert consensus group on congenital heart disease. J Cardiovasc Magn Reson 2013; 15: 51

[16] Schulz-Menger J, Bluemke DA, Bremerich J et al. Standardized image interpretation and post processing in cardiovascular magnetic resonance: Society for Cardiovascular Magnetic Resonance (SCMR) Board of Trustees Task Force on Standardized Post Processing. J Cardiovasc Magn Reson 2013; 15: 35
[17] Anderson TJT, Lu N, Brook OR. Disease-Specific Report Templates for Your Practice. J Am Coll Radiol 2017; 14: 1055-1057

[18] Marcus FI, McKenna W], Sherrill D et al. Diagnosis of arrhythmogenic right ventricular cardiomyopathy/dysplasia: Proposed Modification of the Task Force Criteria. Eur Heart J 2010; 31: 806-814

[19] Friedrich MG, Sechtem U, Schulz-Menger ] et al. Cardiovascular Magnetic Resonance in Myocarditis: A JACC White Paper. J Am Coll Cardiol 2009; 53: 1475-1487

[20] Ferreira VM, Schulz-Menger ], Holmvang G et al. Cardiovascular Magnetic Resonance in Nonischemic Myocardial Inflammation. J Am Coll Cardiol 2018; 72: 3158-3176

[21] Kawel-Boehm N, Maceira A, Valsangiacomo-Buechel ER et al. Normal values for cardiovascular magnetic resonance in adults and children. J Cardiovasc Magn Reson [Internet] Dezember 2015 [zitiert 18. November 2018]; 17(1). Verfügbar unter: http://jcmr-online.com/content/17/1/29

[22] Messroghli DR, Moon JC, Ferreira VM et al. Clinical recommendations for cardiovascular magnetic resonance mapping of $\mathrm{T} 1, \mathrm{~T} 2, \mathrm{~T} 2 *$ and extracellular volume: A consensus statement by the Society for Cardiovascular Magnetic Resonance (SCMR) endorsed by the European Association for Cardiovascular Imaging (EACVI). I Cardiovasc Magn Reson [Internet] Dezember 2017 [zitiert 15. Dezember 2018]; 19(1). Verfügbar unter: https:// jcmr-online.biomedcentral.com/articles/10.1186/s12968-017-0389-8

[23] Ferreira PF, Gatehouse PD, Mohiaddin RH et al. Cardiovascular magnetic resonance artefacts. J Cardiovasc Magn Reson 2013; 15: 41

[24] Araoz P, Glockner J, Mcgee K et al. 3 Tesla MR imaging provides improved contrast in first-pass myocardial perfusion imaging over a range of gadolinium doses. J Cardiovasc Magn Reson 2005; 7: 559-564

[25] Utz W, Niendorf T, Wassmuth R et al. Contrast-dose relation in first-pass myocardial MR perfusion imaging. J Magn Reson Imaging 2007; 25: $1131-1135$

[26] Cerqueira MD, Weissman NJ, Dilsizian V et al. Standardized Myocardial Segmentation and Nomenclature for Tomographic Imaging of the Heart. J Cardiovasc Magn Reson 2002; 4: 203-210

[27] Te Riele ASJM, James CA, Philips B et al. Mutation-Positive Arrhythmogenic Right Ventricular Dysplasia/Cardiomyopathy: The Triangle of Dysplasia Displaced: ARVD/C: The Triangle of Dysplasia Displaced. J Cardiovasc Electrophysiol 2013; 24: 1311-1320

[28] Deutsche Röntgengesellschaft e. V. Nutzungslizenz Befundvorlagen der DRG [Internet]. Verfügbar unter: https://www.befundung.drg.de/ de-DE/3478/creative-commons-lizenz-cc-by-nc-nd/

[29] Assomull RG, Prasad SK, Lyne ] et al. Cardiovascular Magnetic Resonance, Fibrosis, and Prognosis in Dilated Cardiomyopathy. J Am Coll Cardiol 2006; 48: 1977-1985

[30] Kumar S, Van Ness G, Bender A et al. Standardized Goal-Directed Valsalva Maneuver for Assessment of Inducible Left Ventricular Outflow Tract Obstruction in Hypertrophic Cardiomyopathy. J Am Soc Echocardiogr 2018; 31: 791-798

[31] Mahrholdt H, Wagner A, Judd RM et al. Delayed enhancement cardiovascular magnetic resonance assessment of non-ischaemic cardiomyopathies. Eur Heart J 2005; 26: 1461-1474

[32] Noureldin RA, Liu S, Nacif MS et al. The diagnosis of hypertrophic cardiomyopathy by cardiovascular magnetic resonance. ] Cardiovasc Magn Reson 2012; 14: 17

[33] Gotschy A, Saguner AM, Niemann M et al. Right ventricular outflow tract dimensions in arrhythmogenic right ventricular cardiomyopathy/dysplasia - a multicentre study comparing echocardiography and cardiovascular magnetic resonance. Eur Heart J - Cardiovasc Imaging 2018; 19: 516-523

[34] Rastegar N, Te Riele ASJM, James CA et al. Fibrofatty Changes: Incidence at Cardiac MR Imaging in Patients with Arrhythmogenic Right Ventricular Dysplasia/Cardiomyopathy. Radiology 2016; 280: 405-412

[35] Chandarana H, Lim RP, Jensen JH et al. Hepatic Iron Deposition in Patients With Liver Disease: Preliminary Experience With Breath-Hold Multiecho T2*-Weighted Sequence. Am J Roentgenol 2009; 193: 1261-1267 